OPEN ACCESS

Edited by:

Luca Bonfanti,

Università degli Studi di Torino, Italy

Reviewed by:

Philippe Vullioud,

University of Cambridge,

United Kingdom

Joshua Breunig,

Cedars-Sinai Medical Center,

United States

*Correspondence:

Maria K. Oosthuizen

moosthuizen@zoology.up.ac.za

Specialty section This article was submitted to

Neurogenesis,

a section of the journal

Frontiers in Neuroscience

Received: 19 January 2017 Accepted: 16 October 2017

Published: 30 October 2017

Citation:

Oosthuizen MK (2017) From Mice to

Mole-Rats: Species-Specific

Modulation of Adult Hippocampal

Neurogenesis.

Front. Neurosci. 11:602

doi: 10.3389/fnins.2017.00602

\section{From Mice to Mole-Rats: Species-Specific Modulation of Adult Hippocampal Neurogenesis}

\author{
Maria K. Oosthuizen* \\ Department of Zoology and Entomology, University of Pretoria, Pretoria, South Africa
}

Rodent populations living in their natural environments have very diverse ecological and life history profiles that may differ substantially from that of conventional laboratory rodents. Free-living rodents show species-specific neurogenesis that are dependent on their unique biology and ecology. This perspective aims to illustrate the benefit of studying wild rodent species in conjunction with laboratory rodents. African mole-rats are discussed in terms of habitat complexity, social structures, and longevity. African mole-rats are a group of subterranean rodents, endemic to Africa, that show major differences in both intrinsic and extrinsic traits compared to the classical rodent models. Mole-rats exhibit a spectrum of sociality within a single family, ranging from solitary to eusocial. This continuum of sociality provides a platform for comparative testing of hypotheses. Indeed, species differences are apparent both in learning ability and hippocampal neurogenesis. In addition, social mole-rat species display a reproductive division of labor that also results in differential hippocampal neurogenesis, independent of age, offering further scope for comparison. In conclusion, it is evident that neurogenesis studies on conventional laboratory rodents are not necessarily representative, specifically because of a lack of diversity in life histories, uniform habitats, and low genetic variability. The observed level of adult neurogenesis in the dentate gyrus is the result of an intricate balance between many contributing factors, which appear to be specific to distinct groups of animals. The ultimate understanding of the functional and adaptive role of adult neurogenesis will involve research on both laboratory animals and natural rodent populations.

Keywords: African mole-rats, breeding, laboratory rodents, neurogenesis, non-breeding, social, solitary

\section{INTRODUCTION}

It is widely accepted that adult neurogenesis is restricted to two neurogenic regions in the mammalian brain, the subventricular zone of the lateral ventricles and the subgranular zone of the hippocampus (Gage, 2000). Adult hippocampal neurogenesis (AHN) is a dynamic process that has been implicated in hippocampus dependent cognitive functions and both positive and negative regulators of AHN have been described (Aimone et al., 2014). However, the majority of our knowledge originates from studies performed on a few laboratory species that are highly inbred and are maintained in stable laboratory conditions (Kempermann, 2012). Intrinsic and extrinsic traits of wild rodents that were not raised in the laboratory, may differ significantly from that of conventional laboratory animals in factors such as genetic variability, social structure, habitat 
complexity, and longevity, all of which can influence AHN (Kuhn et al., 1996; Kempermann et al., 1997a; Kozorovitskiy and Gould, 2004). Hence, the main drivers of AHN may interact in diverse and unpredictable ways to produce opposite patterns of AHN in wild rodents compared to conventional laboratory rodents, or the drivers may be altogether different.

Since laboratory rodents are frequently used as models for disease-related medical research, it is imperative to understand their limitations and appreciate that life history may influence experimental results. Although the functional significance of AHN may overlap in diverse taxonomic groups, the adaptive value thereof may differ considerably across taxa. Indeed, substantial differences in the extent and magnitude of neurogenesis between mice and humans have been revealed (Jessberger and Gage, 2014). Hence, the investigation of species other than conventional laboratory animals with different traits may provide a useful comparative framework to investigate the adaptive advantage of AHN.

The aim of this work is to highlight that both intrinsic and extrinsic traits of non-conventional rodent species can deviate significantly from that of conventional laboratory animals, and may affect the modulation of AHN very differently depending on species specific requirements. A relevant example is the African mole-rats, a group of subterranean rodents, endemic to Africa. African mole-rats are rodent moles that belong to the family Bathyergidae, which differ radically from laboratory rodents in a number of contexts such as environmental niche, social structures, behavior, and longevity (Figure 1). These factors will be compared between laboratory rodents and mole-rats to provide a perspective on differences in the adaptive value of neurogenesis in the two groups of rodents.

\section{NEUROGENESIS IN CONVENTIONAL LABORATORY RODENT MODELS}

Adult hippocampal neurogenesis (AHN) has been extensively studied in laboratory rodents, both in the context of basal and experimentally manipulated levels of AHN. Both positive and negative regulators of neurogenesis have been identified, some factors are context dependent and may serve as positive, and negative regulators. The factors mentioned below is by no means a complete survey of all potential regulators, merely ones deemed relevant for the ensuing discussion.

\section{Habitat Complexity}

Laboratory animals frequently live in a relatively constant habitat, lacking many of the external factors that can influence their biology. Several studies have demonstrated increased AHN in laboratory rodents in response to enriched environments (Kempermann et al., 1997b, 1998; Nillson et al., 1999; Brown et al., 2003). Habitat complexity increases the need for behavioral flexibility (Amrein, 2015), thus free-living rodent species living in very complex habitats have been shown to exhibit much higher neurogenesis compared to animals that inhabit less complex habitats (Amrein et al., 2007; Garthe et al., 2009; Cavegn et al., 2013).

\section{Social Environment}

The social environment of laboratory animals can exert both positive and negative effects on neurogenesis, depending on the circumstances. Laboratory rodents such as mice and rats are typically communal species and social interactions have been shown to significantly affect the regulation of adult neurogenesis in the hippocampus (Fowler et al., 2008; Lieberwirth and Wang, 2012). Social status can influence the rate of neurogenesis where, in laboratory rodents, animals with a higher status typically show more neurogenesis than the ones with a lower social status (Gould et al., 1997, 1998; Kozorovitskiy and Gould, 2004; Thomas et al., 2007; Wu et al., 2014). Variation in estrogen levels may be the underlying mechanism responsible for differences in AHN between dominant and subordinate animals. Dominant animals typically have a higher probability of breeding, and breeding individuals usually exhibit higher levels of estrogen. Estrogen has been shown to play a role in proliferation, the survival as well as the activation of the new neurons (Fowler et al., 2008). Following ovariectomy, AHN was reduced but this could be reversed by estrogen replacement (Tanapat et al., 1999). Cell proliferation in the dentate gyrus (DG) of the hippocampus of laboratory rat females also fluctuates according to the estrous cycle, with higher cell proliferation when more estrogen is present (Tanapat et al., 1999).

Stress hormones can have positive or negative effects on $\mathrm{AHN}$, depending on the type of stressor and whether the stress is acute or chronic (Schoenfeld and Gould, 2012). High levels of corticosterone in response to social isolation causes a reduction in neurogenesis and also decreases performance in other behavioral tests (Stranahan et al., 2006). This effect seems to be larger in females compared to males (Westenbroek et al., 2004). In contrast, exercise (running) induces lower levels of corticosterone and is associated with an increase in $\mathrm{AHN}$ (Stranahan et al., 2006).

\section{Age}

Age is commonly viewed as a potent negative regulator of $\mathrm{AHN}$ since there is a dramatic decline in neurogenesis that appears to be age related in most mammals investigated, laboratory rodents included (Kuhn et al., 1996; Amrein et al., 2004; Ben Abdallah et al., 2010). This downregulation of neurogenesis is not correlated with the environment or other species-specific traits such as longevity or developmental strategy (Amrein et al., 2004). Technically, this decrease occurs relatively early in life, after which the level of neurogenesis remains relatively stable.

\section{Cognitive Activity}

A vast number of studies suggest a link between learning, memory, and adult neurogenesis. Initial studies showed that learning increases hippocampal neurogenesis (Gould et al., 1999a,b) which accordingly enhances spatial memory (Snyder et al., 2005; Winocur et al., 2006). In turn, learning impairments are associated with a reduction in hippocampal neurogenesis (Lemaire et al., 2000). However, more recent evidence suggests that this is highly species and context dependent and the results are not always consistent (Dobrossy et al., 2003; Jaholkowski et al., 2009; Groves et al., 2013; Duarte-Guterman et al., 2015). 


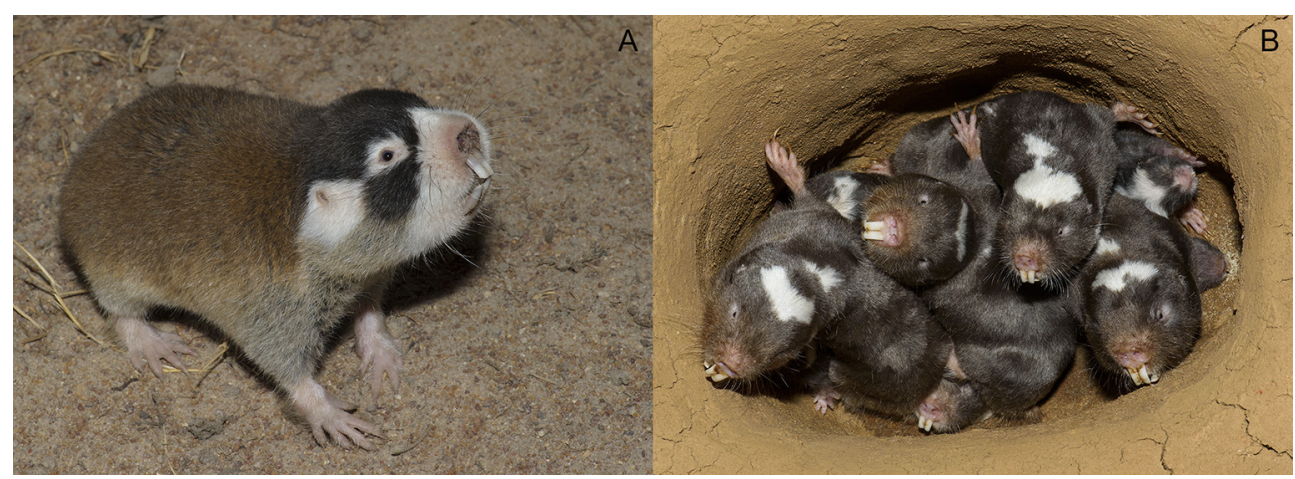

FIGURE 1 | (A) Solitary Cape mole-rat. (B) A colony of social Damaraland mole-rats.

In some cases, neurogenesis has no effect on spatial memory at all (Groves et al., 2013). The mixed results may be attributed to the type of behavioral tests performed or the history and age of the animals, or a combination of factors.

\section{COMPARING LABORATORY MODELS WITH THE MOLE-RAT MODEL}

Some factors that can influence AHN in rodents exhibit parallel features in laboratory rodent models and the mole-rat model, with the difference that it occurs naturally in mole-rats but are human induced in laboratory rodents. Other factors are distinctly different for the two models.

\section{Habitat Complexity}

Habitat complexity, or the lack thereof, can influence AHN in rodents depending on the need for behavioral flexibility (Amrein, 2015). Laboratory rodents have probably adapted to their relatively uniform and confined laboratory habitat over many generations in captivity, with accompanying alterations in behavioral needs (Toth et al., 2011). Although in a different spatial context, the sealed burrow systems of mole-rats is devoid of light and lack many other sensory cues available to aboveground living rodents (Burda et al., 1990). Mole-rats thus present a model that naturally inhabits a uniform environment. In addition, even the simplistic environment of mole-rats shows interspecific variation in terms of length and complexity of the tunnels, depending on the social structure of the species, presenting opportunity for comparisons.

\section{Social Structure}

Laboratory mice and rats are generally classified as social and polygamous but the social structure is not rigidly fixed (Lund, 1975; Hedrich, 2012). As a taxonomic group, molerats show much more diverse and complex social organizations compared to laboratory animals. Bathyergids exhibit a spectrum of sociality within a single taxonomic family, ranging from strictly solitary to highly social species (Faulkes et al., 1997). Solitary species are typically polygamous whereas social species tend to be more monogamous. Social mole-rats live in family groups that exhibit a distinct reproductive division of labor (Jarvis, 1981; Bennett, 1988). Reproduction is restricted to a single female and one or two males, while the remainder of the colony comprises overlapping generations of subordinate animals that are reproductively suppressed. Mole-rats breed cooperatively, and the non-breeding individuals assist with the rearing of offspring and maintenance of the tunnel system (Jarvis, 1981; Bennett, 1988). Social mole-rat colonies exhibit linear dominance hierarchies where larger animals are dominant over smaller animals (Jacobs et al., 1991), but breeding animals are always dominant over non-breeding animals. In other rodents, both dominance and reproductive status have been shown to influence AHN (Tanapat et al., 1999; Kozorovitskiy and Gould, 2004). Therefore, the difference in social structures between species and the within species status differences in the mole-rat model provide abundant opportunities for empirical testing of predictions in a comparative setting.

\section{Age and Longevity}

Laboratory mice and rats have maximum lifespans of under 5 years (Gorbunova et al., 2008), whereas their free-living counterparts may have a much shorter life expectancy. Similar sized mole-rats, especially the social species, can attain ages three to six-fold that of laboratory animals. In captivity, the age of 16 years have been recorded for social Fukomys mole-rats (Dammann et al., 2011) and the age of 32 years for naked molerats (Heterocephalus glaber) (Buffenstein and Jarvis, 2002). An exponential decline in AHN is apparent in both long and shortlived species, but the slower maturation of longer lived species may offer a larger window for experimental manipulation of the baseline AHN.

\section{MOLE-RAT NEUROGENESIS}

Morphologically, the dentate gyrus of mole-rats is comparatively smaller than that of other rodents, with fewer granule cells (Amrein et al., 2014). Mole-rats in general have very low levels of neurogenesis in the hippocampus (Amrein et al., 2014; Penz et al., 2015; Oosthuizen and Amrein, 2016). Normalized proliferating cell numbers of mole-rats are comparable with that 
of other rodents however the normalized young neurons are lower (Amrein et al., 2014).

\section{Habitat Complexity}

The lower survival rate of young neurons in mole-rats supports a habitat-dependent modulation of neurogenesis. The habitat complexity of mole-rat burrow systems is very low when compared to the highly complex three-dimensional environments of surface dwelling rodents. The sealed burrow systems of mole-rats lack external cues and therefore present a very homogenous and stable habitat (Burda et al., 1990).

Despite the overall low habitat complexity, the length and complexity of mole-rat burrows differ between species, it depends on a number of factors including sociality, habitat type, resource availability, and population density (Le Comber et al., 2002). The burrow systems of Damaraland mole-rats (Fukomys damarensis) can reach up to $2 \mathrm{~km}$ in length (Bennett and Faulkes, 2000), while burrow systems of solitary species such as the Cape molerat (Georychus capensis) are generally much shorter (Thomas et al., 2012). Within the context of the subterranean niche, interspecies differences in hippocampal neurogenesis that is consistent with the relative length and complexity of the burrows of the different species, is evident. The social and solitary species have similar numbers of granule cells despite size differences in the species [Cape: 150-200 g; Highveld (Cryptomys hottentotus pretoriae; intermediately social species): 80-120 g; Damaraland: 120-150 g; Bennett and Faulkes, 2000, pers. obs.], however both the Highveld and Damaraland mole-rats have more proliferating cells compared to Cape mole-rats (Ki67 staining) (Amrein et al., 2014; Oosthuizen and Amrein, 2016). The numbers of young neurons show large within species variation, thus young neuron numbers of the individual species show some overlap.

\section{Social Status}

Despite the low rate of neurogenesis, a status dependent amount of hippocampal neurogenesis is evident in the social Damaraland mole-rats. Seemingly in contrast with results from laboratory rodents, the breeding females, or queens, have lower numbers of both proliferating cells and young neurons compared to subordinate colony members (Oosthuizen and Amrein, 2016) (Figure 2). A similar occurrence is observed in the naked mole-rat, where breeding animals were found to have significantly less young neurons, as visualized by doublecortin (DCX) immunoreactive neurons, compared to the non-breeding animals (Peragine et al., 2014). Differential AHN in breeding and non-breeding mole-rats may potentially have an endocrinological basis. Both reproductive hormones and stress hormones have been shown to modulate neurogenesis in laboratory rodents (Cameron and Gould, 1994; Gould and Tanapat, 1999; Tanapat et al., 1999).

In highly social species such as the Damaraland mole-rat and the naked mole-rat, the breeding females have higher estrogen levels compared to non-breeding animals (Bennett and Jarvis, 1988; Faulkes et al., 1990; Bennett, 1994), thus one would also expect an upregulation of neurogenic cells, yet the opposite is true (Peragine et al., 2014; Oosthuizen and Amrein, 2016). In the case of mole-rats, estrogen appears to rather downregulate AHN. Similarly, cell proliferation is inhibited in the dentate gyrus of female meadow voles in the breeding season, when high estrogen levels are present, compared to females out of the breeding season, although more cells survive in the reproductively active females (Galea and McEwen, 1999; Ormerod and Galea, 2001). A potential mechanism for the disparity in the effect of estrogen on AHN may be related to the density of the estrogen receptor $\alpha$ in the dentate gyrus. In voles, estrogen increased the density of the estrogen receptor $\alpha(\mathrm{ER} \alpha)$ (Fowler et al., 2005), however this increase was region specific and no difference was observed in the dentate gyrus. In mole-rats, non-breeding Damaraland mole-rat females express lower levels of ER $\alpha$ compared to the breeders in brain regions important for reproduction (Voigt et al., 2014). The density of the ER $\alpha$ has not been investigated in the DG of mole-rats thus far, but could potentially also show differential expression between breeder and non-breeder mole-rats. In naked mole-rats, no significant relationships could

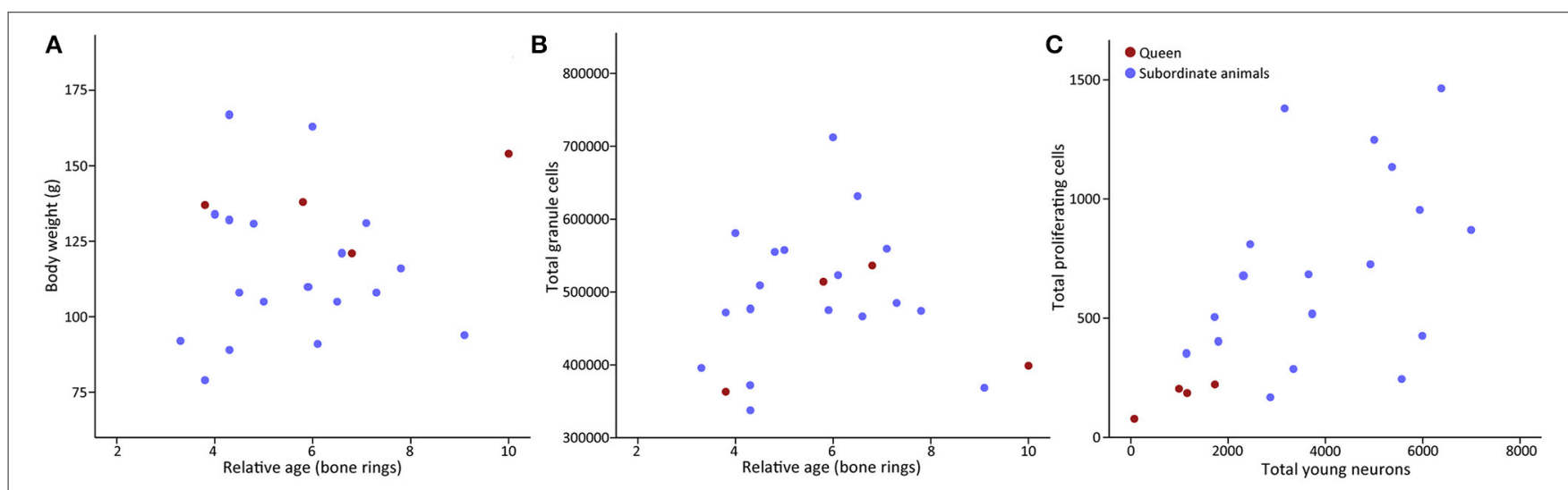

FIGURE 2 | Scatter plots of relative age $\mathrm{x}$ body weight, total granule cells $\times$ relative age, and proliferation $\times$ young neurons in Damaraland mole-rats. (A) The body weight of Damaraland mole-rats does not increase with relative age, (B) total granule cells remains stable with relative age, and (C) a scatter plot of proliferating cells (Ki67) and young neurons (PSA-NCAM) in dominant and subordinate Damaraland mole-rats (Modified from (Oosthuizen and Amrein, 2016), with permission from Elsevier). 
be identified between circulating gonadal steroids and DCX (Peragine et al., 2014), however this do not exclude potential differences in proliferating cells or receptors for the hormones.

Stress hormones have not been exhaustively investigated in mole-rats in terms of social status in the colony. It appears that when the social structure is stable, there is no difference cortisol levels between breeding and non-breeding animals (Clarke and Faulkes, 1998; Clarke et al., 2001), but instability in the social structure is associated with increased cortisol levels in nonbreeding animals compared to the breeding animals (Clarke and Faulkes, 1997). In congruence with this, a higher concentration of CRF receptor binding sites is present in non-breeding naked mole-rats (Beery et al., 2016). In addition, CRF receptor binding sites also differ between solitary and social mole-rat species (Coen et al., 2015), where differences in proliferating cells have been shown. Stress hormones are also expressed in response to running and exercise, where it is associated with an upregulation of AHN (Stranahan et al., 2006). Non-breeding Damaraland mole-rat females show higher levels of locomotor activity than the breeding females (Oosthuizen and Bennett, 2015), therefore increased activity could, at least in part, explain the higher AHN in non-breeding mole-rats.

\section{Age and Longevity}

In agreement with their increased life expectancies, mole-rats have long gestation times and subsequent postnatal development is slower than other rodents. Like other animals, mole-rats also show a steep decline in neurogenesis at a relatively young age (Penz et al., 2015). A slow postnatal development may increase the expected window for higher levels of neurogenesis seen in all young animals between birth and puberty (Penz et al., 2015). In mole-rats, puberty is reached around the age of 1 year, when neurogenesis should start to decline exponentially (Bennett and Faulkes, 2000; Penz et al., 2015), however this has not been tested empirically.

\section{Cognitive Activity}

As a result of their lightless environment, subterranean animals rely on other mechanisms such as tactile stimuli and memory to navigate their burrow systems (du Toit et al., 2012). Despite the relatively uniform habitat of the sealed tunnel systems, the difference in tunnel length and complexity appears to be sufficient stimulation to induce learning differences in the different species. Damaraland mole-rats with longer and more complex burrow systems, show superior learning abilities

\section{REFERENCES}

Aimone, J. B., Li, Y., Lee, S. W., Clemenson, G. D., Deng, W., and Gage, F. H. (2014). Regulation and function of adult neurogenesis: from genes to cognition. Physiol. Rev. 94, 991-1026. doi: 10.1152/physrev.00004.2014

Amrein, I. (2015). Adult hippocamal neurogenesis in natural populations of mammals. Cold Spring Harb. Perspect. Biol. 7:a021295. doi: 10.1101/ cshperspect.a021295

Amrein, I., Becker, A. S., Engler, S., Huang, S. H., Muller, J., Slomianka, L., et al. (2014). Adult neurogenesis and its anatomical context in the hippocampus of three mole-rat species. Front. Neuroanat. 8:39. doi: 10.3389/fnana.2014.00039 compared to the solitary Cape mole-rat (Costanzo et al., 2009; Oosthuizen et al., 2013), Oosthuizen, unpublished data). The enhanced learning abilities of Damaraland mole-rats are associated with more proliferating cells compared to Cape mole-rats (Amrein et al., 2014; Oosthuizen and Amrein, 2016). Although the basal level of neurogenesis differs with social status in highly social species, there is no corresponding difference in learning abilities.

\section{CONCLUSIONS}

Neurogenesis studies performed on conventional laboratory rodents by far outnumber those on wild species. Research on laboratory animals is necessary as laboratory models are very useful and convenient tools for the fundamental understanding of the molecular basis and the regulation of neurogenesis, however they have limitations. Laboratory animals live in a completely uniform habitat without natural predators and are bred to minimize variability. To comprehend the functional and adaptive significance of neurogenesis, the environmental, genetic and physiological variation of natural populations is essential. It is important to appreciate that the adaptive value of neurogenesis is species specific. In many instances, natural animal populations show very different and more varied physiological and neurological responses compared to their laboratory counterparts.

This perspective primarily illustrates the diversity in environmental conditions, social structures and longevity in rodent species. Animal models that display a different set of species-specific features may provide insight into the functional and adaptive significance of adult neurogenesis. Mole-rats differ from conventional laboratory animals in a number of important ways, specifically social structure and longevity. Mole-rats (and indeed other natural rodent populations) are not suggested as a replacement for conventional laboratory rodents in neurogenesis research, but rather to complement the existing body of information. Ultimately, understanding the functional and adaptive context of adult neurogenesis will require research on both laboratory animals and natural rodent populations.

\section{AUTHOR CONTRIBUTIONS}

The author confirms being the sole contributor of this work and approved it for publication. 
Ben Abdallah, N. M., Slomianka, L., Vyssotski, A. L., and Lipp, H. P. (2010). Early age-related changes in adult hippocampal neurogenesis in C57 mice. Neurobiol. Aging 31, 151-161. doi: 10.1016/j.neurobiolaging.2008.03.002

Bennett, N. C. (1988). The Trend Towards Sociality in Three Species of Southern African Mole-Rats Bathyergidae: Causes and Consequences. Ph.D., University of Cape Town.

Bennett, N. C. (1994). Reproductive suppression in social Cryptomys damarensis colonies - a lifetime of socially-induced sterility in males and females (Rodentia: Bathyergidae). J. Zool. Lond. 234, 25-39. doi: 10.1111/j.1469-7998. 1994.tb06054.x

Bennett, N. C., and Faulkes, C. G. (2000). African Mole-Rats: Ecology and Eusociality. Cambridge, UK: Cambridge University Press.

Bennett, N. C., and Jarvis, J. U. (1988). The social structure and reproductive biology of colonies of the mole-rat, Cryptomys damarensis (Rodentia, Bathyergidae). J. Mammal. 69, 293-302. doi: 10.2307/1381379

Brown, J., Cooper-Kuhn, C. M., Kempermann, G., Van Praag, H., Winkler, J., Gage, F. H., et al. (2003). Enriched environment and physical activity stimulate hippocampal but not olfactory bulb neurogenesis. Eur. J. Neurosci. 17, 2042-2046. doi: 10.1046/j.1460-9568.2003.02647.x

Buffenstein, R., and Jarvis, J. U. (2002). The naked mole-rat - a new record for the oldest living rodent. Sci. Aging Knowl. Environ. 21:7. doi: 10.1126/sageke.2002.21.pe7

Burda, H., Bruns, V., and Muller, J. (1990). Evolution of Subterranean Mammals a the Organismal and Molecular Levels. New York, NY: Wiley.

Cameron, H.A., and Gould, E. (1994). Adult neurogenesis is regulated by adrenal steroids in the dentate gyrus. Neuroscience 612, 203-209.

Cavegn, N., van Dijk, R. M., Menges, D., Brettschneider, H., Phalanndwa, M., Chimimba, C. T., et al. (2013). Habitat-specific shaping of proliferation and neuronal differentiation in adult hippocampal neurogenesis of wild rodents. Front. Neurosci. 7:59. doi: 10.3389/fnins.2013.00059

Clarke, F. M., and Faulkes, C. G. (1997). Dominance and queen succession in captive colonies of the eusocial naked mole-rat, Heterocephalus glaber. Proc. $R$. Soc. Lond. 264, 993-1000. doi: 10.1098/rspb.1997.0137

Clarke, F. M., and Faulkes, C. G. (1998). Hormonal and behavioural corelates of male dominance and reproductive status in captive colonies of the naked mole-rat, Heterocephalus glaber. Proc. R. Soc. Lond. B. 265, 1391-1399. doi: 10.1098/rspb.1998.0447

Clarke, F. M., Miethe, G. H., and Bennett, N. C. (2001). Reproductive suppression in female Damaraland mole-rats Cryptomys damarensis: dominant control or self-restraint? Proc. R. Soc. Lond. 268, 899-909. doi: 10.1098/rspb.2000.1426

Coen, C. W., Kalamatianos, T., Oosthuizen, M. K., Poorun, R., Faulkes, C. G., and Bennett, N. C. (2015). Sociality and the telencephalic distribution of corticotrophin-releasing factor, urocortin 3 , and binding sites for CRF type 1 and type 2 receptors: a comparative study of eusocial naked mole-rats and solitary Cape mole-rats. J. Comp. Neurol. 523, 2344-2371. doi: 10.1002/cne.23796

Costanzo, M. S., Bennett, N. C., and Lutermann, H. (2009). Spatial learning and memory in African mole-rats: the role of sociality and sex. Physiol. Behav. 96, 128-134. doi: 10.1016/j.physbeh.2008.09.008

Dammann, P., Sumbera, R., Masmann, C., Scherag, A., and Burda, H. (2011). Extended longevity of reproductives appears to be common in Fukomys mole-rats (Rodentia, Bathyergidae). PLoS ONE 6:e18757. doi: 10.1371/journal.pone.0018757

Dobrossy, M. D., Drapeau, E., Aurousseau, C., Le Moal, M., Piazza, P. V., and Abrous, D. N. (2003). Differential effects of learning on neurogenesis: learning increases or decreases the number of newly born cells depending on their birth date. Mol. Psychiatry 8, 974-982. doi: 10.1038/sj.mp.4001419

du Toit, L., Bennett, N. C., Nickless, A., and Whiting, M. J. (2012). Influence of spatial environment on maze learning in an African mole-rat. Anim. Cogn. 15, 797-806. doi: 10.1007/s10071-012-0503-0

Duarte-Guterman, P., Yagi, S., Chow, C., and Galea, L. A. (2015). Hippocampal learning, memory, and neurogenesis: effects of sex and estrogens across the lifespan in adults. Horm. Behav. 74, 37-52. doi: 10.1016/j.yhbeh.2015. 05.024

Faulkes, C. G., Abbott, D. H., and Jarvis, J. U. M. (1990). Social suppresion of ovarian cyclicity in captive and wild colonies of naked mole-rats, Heterocephalus glaber. J. Reprod. Fert. 88, 559-568. doi: 10.1530/jrf.0.08 80559
Faulkes, C. G., Bennett, N. C., Bruford, M. W., O’Brien, H. P., Aguilar, G. H., and Jarvis, J. U. (1997). Ecological constraints drive social evolution in the African mole-rats. Proc. Biol. Sci. 264, 1619-1627.

Fowler, C. D., Johnson, F., and Wang, Z. (2005). Estrogen regulation of cell proliferation and distribution of estrogen receptor-alpha in the brains of adult female prairie and meadow voles. J. Comp. Neurol. 489, 166-179. doi: $10.1002 /$ cne. 20638

Fowler, C. D., Liu, Y., and Wang, Z. (2008). Estrogen and adult neurogenesis in the amygdala and hypothalamus. Brain Res. Rev. 57, 342-351. doi: 10.1016/ j.brainresrev.2007.06.011

Gage, F. H. (2000). Mammalian neural stem cells. Science 287:1433. doi: $10.1126 /$ science.287.5457.1433

Galea, L. A. M., and McEwen, B. S. (1999). Sex and seasonal differences in the rate of cell proliferation in the dentate gyrus of adult wild meadow voles. Neuroscience 89, 955-964. doi: 10.1016/S0306-4522(98)00345-5

Garthe, A., Behr, J., and Kempermann, G. (2009). Adult-generated hippocampal neurons allow the flexible use of spatially precise learning strategies. PLOS ONE 4:e5464. doi: 10.1371/journal.pone.0005464

Gorbunova, V., Bozzella, M. J., and Seluanov, A. (2008). Rodents for comparative aging studies: from mice to bevers. Age 30, 111-119. doi: 10.1007/s11357-008-9053-4

Gould, E., and Tanapat, P. (1999). Stress and hippocampal neurogenesis. Biol. Pshychiatry 46, 1472-1479. doi: 10.1016/S0006-3223(99)00247-4

Gould, E., Beylin, A., Tanapat, P., Reeves, A., and Shors, T. J. (1999a). Learning enhances adult neurogenesis in the hippocampal formation. Nat. Neurosci. 2, 260-265.

Gould, E., McEwen, B. S., Tanapat, P., Galea, L. A. M., and Fuchs, E. (1997). Neurogenesis in the dentate gyrus of the adult tree shrew is regulated by psychosocial stress and NMDA receptor activation. J. Neurosci. Methods 17, 2492-2498.

Gould, E., Tanapat, P., Hastings, N. B., and Shors, T. J. (1999b). Neurogenesis in adulthood: a possible role in learning. Trends in Cogn. Sci. 3, 186-192.

Gould, E., Tanapat, P., McEwen, B., Flügge, G., and Fuchs, E. (1998). Proliferation of granule cell precursors in the dentate gyrus of adult monkeys is diminished by stress. Proc. Natl. Acad. Sci. U.S.A. 95, 3168-3171. doi: $10.1073 /$ pnas.95.6.3168

Groves, J. O., Leslie, I., Huang, G. J., McHugh, S. B., Taylor, A., Mott, R., et al. (2013). Ablating adult neurogenesis in the rat has no effect on spatial processing: evidence from a novel pharmacogenetic model. PLoS Genet 9:e1003718. doi: 10.1371/journal.pgen.1003718

Hedrich, H. J. (2012). The Laboratory Mouse. London, UK: Academic Press.

Jacobs, D. S., Bennett, N. C., Jarvis, J. U. M., and Crowe, T. M. (1991). The colony structure and dominance hierarchy of the Damaraland mole-rat, Cryptomys damarensis (Rodentia: Bathyergidae), from Namibia. J. Zool. Lond. 224, 553-576. doi: 10.1111/j.1469-7998.1991.tb03785.x

Jaholkowski, P., Kiryk, A., Jedynak, P., Ben Abdallah, N. M., Knapska, E., Kowalczyk, A., et al. (2009). New hippocampal neurons are not obligatory for memory formation; cyclin D2 knockout mice with no adult brain neurogenesis show learning. Learn. Mem. 16, 439-451. doi: 10.1101/lm.1459709

Jarvis, J. U. M. (1981). Eusociality in a mammal - cooperative breeding in the naked mole-rat Heterocephalus glaber colonies. Science 212, 571-573. doi: $10.1126 /$ science. 7209555

Jessberger, S., and Gage, F. H. (2014). Adult neurogenesis: bridging the gap between mice and humans. Trends Cell Biol. 24, 558-563. doi: 10.1016/j.tcb.2014.07.003

Kempermann, G. (2012). New neurons for 'survival of the fittest. Nat. Rev. Neurosci. 13, 727-736. doi: 10.1038/nrn3319

Kempermann, G., Brandon, E. P., and Gage, F. H. (1998). Environmental stimulation of $129 / \mathrm{SvJ}$ mice causes increased cell proliferation and neurogenesis in the adult dentate gyrus. Curr. Biol. 8, 939-942. doi: 10.1016/ S0960-9822(07)00377-6

Kempermann, G., Kuhn, H. G., and Gage, F. H. (1997a). Genetic influence on neurogenesis in the dentate gyrus of adult mice. Proc. Natl. Acad. Sci. U.S.A. 94, 10409-10414.

Kempermann, G., Kuhn, H. G., and Gage, F. H. (1997b). More hippocampal neurons in adult mice living in an enriched environment. Nature 386, 493-495.

Kozorovitskiy, Y., and Gould, E. (2004). Dominance hierarchy influences adult neurogenesis in the dentate gyrus. J. Neurosci. 24, 6755-6759. doi: 10.1523/JNEUROSCI.0345-04.2004 
Kuhn, H. G., Dickenson-Anson, H., and Gage, F. H. (1996). Neurogenesis in the dentate gyrus of the adult rat: age-related decrease of neuronal progenitor proliferation. J. Neurosci. 16, 2027-2033.

Le Comber, S. C., Spinks, A. C., Bennett, N. C., Jarvis, J. U. M., and Faulkes, C. G. (2002). Fractal dimension of African mole-rat burrows. Canad. J. Zool. 80, 436-441. doi: 10.1139/z02-026

Lemaire, V., Koehl, M., Le Moal, M., and Abrous, D. N. (2000). Prenatal stress produces learning deficits associated with an inhibition of neurogenesis in the hippocampus. PNAS 97, 11032-11037. doi: 10.1073/pnas.97.20.11032

Lieberwirth, C., and Wang, Z. (2012). The social environment and neurogenesis in the adult mammalian brain. Front. Hum. Neurosci. 6:118. doi: $10.3389 /$ fnhum. 2012.00118

Lund, M. (1975). Social mechanisms and social structure in rats and mice. Ecol. Bull. 19, 255-260.

Nillson, M., Perfilieva, E., Johansson, U., Orwar, O., and Eriksson, P. (1999). Enriched environment increases neurogenesis in the adult rat dentate gyrus and improves spatial memory. J. Neurobiol. 39, 569-578. doi: 10.1002/(SICI)10974695(19990615)39:4<569::AID-NEU10>3.0.CO;2-F

Oosthuizen, M. K., and Amrein, I. (2016). Trading new neurons for status: Adult hippocampal neurogenesis in eusocial Damaraland mole-rats. Neuroscience 324, 227-237. doi: 10.1016/j.neuroscience.2016.03.020

Oosthuizen, M. K., and Bennett, N. C. (2015). The effect of ambient temperature on locomotor activity patterns in reproductive and non-reproductive female Damaraland mole-rats. J. Zool. 297, 1-8. doi: 10.1111/jzo.12254

Oosthuizen, M. K., Scheibler, A. G., Bennett, N. C., and Amrein, I. (2013). Effects of laboratory housing on exploratory behaviour, novelty discrimination and spatial reference memory in a subterranean, solitary rodent, the Cape mole-rat (Georychus capensis). PLoS ONE 8:e75863. doi: 10.1371/journal.pone.0075863

Ormerod, B. K., and Galea, L. (2001). Reproductive status influences cell proliferation and cell survival in the dentate gyrus of adult female meadow voles: a possible regulatory role for estradiol. Neuroscience 102, 369-379. doi: 10.1016/S0306-4522(00)00474-7

Penz, O. K., Fuzik, J., Kurek, A. B., Romanov, R., Larson, J., Park, T. J., et al. (2015). Protracted brain development in a rodent model of extreme longevity. Sci. Rep. 5:11592. doi: 10.1038/srep11592

Peragine, D. E., Simpson, J. A., Mooney, S. J., Lovern, M. B., and Holmes, M. M. (2014). Social regulation of adult neurogenesis in a eusocial mammal. Neuroscience 268, 10-20. doi: 10.1016/j.neuroscience.2014.02.044

Schoenfeld, T. J., and Gould, E. (2012). Stress, stress hormones, and adult neurogenesis. Exp. Neurol. 233, 12-21. doi: 10.1016/j.expneurol.2011.01.008

Snyder, J. S., Hong, N. S., McDonald, R. J., and Wojtowicz, J. M. (2005). A role for adult neurogenesis in spatial long-term memory. Neuroscience 130, 843-852. doi: 10.1016/j.neuroscience.2004.10.009
Stranahan, A. M., Khalil, D., and Gould, E. (2006). Social isolation delays the positive effects of running on adult neurogenesis. Nat. Neurosci. 9, 526-533. doi: $10.1038 / \mathrm{nn} 1668$

Tanapat, P., Hastings, N. B., Reeves, A., and Gould, E. (1999). Estrogen stimulates a transient increase in the number of new neurons in the dentate gyrus of the adult female rat. J. Neurosci. 19, 5792-5801.

Thomas, H. G., Bateman, P. W., Scantlebury, M., Bennett, N. C., and Hayssen, V. (2012). Season but not sex influences burrow length and complexity in the nonsexually dimorphic solitary Cape mole-rat (Rodentia: Bathyergidae). J. Zool. 288, 214-221. doi: 10.1111/j.1469-7998.2012.00944.x

Thomas, R. M., Hotsenpiller, G., and Peterson, D. A. (2007). Acute Psychosocial stress reduces cell survival in adult hippocampal neurogenesis without altering proliferation. J. Neurosci. 27, 2734-2743. doi: 10.1523/JNEUROSCI. 3849-06.2007

Toth, L. A., Kregel, K., Leon, L., and Musch, T. I. (2011). Environmental enrichment of laboratory rodents: the answer depends on the question. Comp. Med. 61, 314-321.

Voigt, C., Gahr, M., Leitner, S., Lutermann, H., and Bennett, N. C. (2014). Breeding status and social environment differentially affect the expression of sex steroid receptor and aromatase mRNA in the brain of female Damaraland mole-rats. Front. Zool. 11:38. doi: 10.1186/1742-9994-11-38

Westenbroek, C., Den Boer, J. A., Veenhuis, M., and Ter Horst, G. J. (2004) Chronic stress and social housing differentially affect neurogenesis in male and female rats. Brain Res. Bull. 64, 303-308. doi: 10.1016/j.brainresbull.2004. 08.006

Winocur, G., Wojtowicz, J. M., Sekeres, M., Snyder, J. S., and Wang, S. (2006). Inhibition of neurogenesis interferes wit hhippicampus-dependent memory function. Hippocampus 16, 296-304. doi: 10.1002/hipo.20163

Wu, M. V., Shamy, J. L., Bedi, G., Choi, C.-W., Wall, M. M., Arango, V., et al. (2014). Impact of social status and antidepressant treatment on neurogenesis in the baboon hippocampus. Neuropsychopharmacology 39, 1861-1871. doi: 10.1038/npp.2014.33

Conflict of Interest Statement: The author declares that the research was conducted in the absence of any commercial or financial relationships that could be construed as a potential conflict of interest.

Copyright (C) 2017 Oosthuizen. This is an open-access article distributed under the terms of the Creative Commons Attribution License (CC BY). The use, distribution or reproduction in other forums is permitted, provided the original author(s) or licensor are credited and that the original publication in this journal is cited, in accordance with accepted academic practice. No use, distribution or reproduction is permitted which does not comply with these terms. 